\title{
Natural pigment of red-fleshed Pitaya (Hylocereus polyrhizus) as dental plaque disclosing agent- A preliminary study
}

\author{
Amaliya Amaliya' (D), Regi Taufik Firdaus ${ }^{2}$ (D), Nunung Rusminah' (D) \\ 'Universitas Padjadjaran, Dental Faculty, Department of Periodontology, Bandung, Indonesia \\ ${ }^{2}$ Universitas Padjadjaran, Dental Faculty, Undergraduate Programme, Bandung, Indonesia
}

ORCID IDs of the authors: A.A. 0000-0002-9164-0542; R.T.F. 0000-0001-7076-7272; N.R. 0000-0002-3709-8625

Cite this article as: Amaliya, A., Firdaus, R. T., \& Rusminah, N. (2020). Natural pigment of red-fleshed Pitaya (Hylocereus polyrhizus) as dental plaque disclosing agent- A preliminary study. Istanbul Journal of Pharmacy, 50 (3), 256-261.

\begin{abstract}
Background and Aims: Dental plaque as the main etiological factor for caries and periodontal disease is transparent, colorless and not easily visible. Visualized dental plaque can be an aid for patients to develop an efficient system of plaque removal. Several disclosing agents have been developed and applied to clinical and home-care use, yet people are still searching for a natural coloring agent which could be considered safe for long term use.

Methods: In this study, various concentrations of Pitaya flesh extract were tested to stain dental plaque taken from 30 volunteers. A disclosing agent of basic-fuchsin already marketed was used as a positive control. The dental plaque specimens were divided into 5 groups and each group was randomly stained either with $0.05 \mathrm{~mL}$ of basic fuchsin as a positive control, or $25 \%$, $50 \%, 75 \%$, or $100 \%$ Pitaya flesh extract. The specimens were then analyzed using a spectrophotometer.

Results: The results showed that Pitaya flesh extract exhibited dental plaque staining ability. The concentration of $75 \%$ Pitaya was similar to the staining ability of basic-fuchsin, while $100 \%$ was superior to that of basic-fuchsin.

Conclusion: Under the limitations of this study, 75\% and 100\% extract of pitaya flesh exhibited dental plaque staining in vitro as assessed by a spectrophotometer.
\end{abstract}

Keywords: Natural pigment, Pitaya, dental plaque

\section{INTRODUCTION}

Dental plaque is the etiological agent for major dental diseases such as caries and periodontal disease. Plaque accumulation may increase under certain conditions, such as poor oral hygiene and inappropriate dietary behavior. When left uncontrolled, following adhesion to the tooth surface, dental plaque grows and reaches maturation and leads to these potential consequences; inflammatory changes on the periodontium resulting in destruction of tissues and loss of attachment or irreversible solubilization of tooth mineral by acid produced by certain bacteria in dental plaque (Seneviratne, Zhang, \& Samaranayake, 2011; Broadbent, Thomson, Boyens, \& Poulton, 2011).

Dental plaque is a biofilm, formed over the hard surfaces in the oral cavity, including calculus and restorations or prosthetic appliances placed in the oral cavity. It comprises living bacterial communities, embedded in a matrix of polymers of host and bacterial origins (Marsh 2010). Mechanical plaque control procedures such as brushing and flossing are effective in reducing plaque, but plaque is rarely removed completely using these measures (Flemmig \& Beikler 2011). Since dental plaque is transparent, colorless, and not easily visible, an individual is not generally aware of the amount or the location of dental plaque in his oral cavity. Therefore, it is necessary 
to detect plaque-covered areas in the oral cavity by using disclosing solutions. Clearly seen plaque can help patients develop an efficient system of plaque removal (Chetrus \& Ion, 2013).

Disclosing solutions work by changing the color of dental plaque so that it contrasts with the tooth surface. Several agents have been incorporated in dentistry, from iodine and erythrosine dye, to a fluorescence and three tone dye that are able to disclose and differentiate between early plaque formation and matured ones (Jayanthi, Shilpapriya, Reddy, Elangovan, Sakthivel, \& Vijayakumar, 2015; Datta, Kumar, Narayanan, Selvamary, \& Sujatha, 2017). However, in the majority of cases, these colorants have disadvantages such as an unpleasant flavor causing subjects to strongly dislike it. In this sense, natural colorants have been suggested as alternatives to the use of synthetic colorants to promote dental hygiene and a patient's motivation. In addition, some artificial colorants caused allergic reactions, or acute toxicity and genetic potential in experimental animals (Miyachi \&Tsutsui, 2005; Sania, Aggarwal, \& Chaubey, 2016). These disadvantages have driven the replacement of synthetic colorants with natural-derived alternatives.

Pitaya or Pitahaya, a fruit native to Mesoamerica, or also called Dragon Fruit or Buah Naga in Indonesia, gathered much interest due to its attractive appearance and taste, as well as nutritional contents (Ortiz-Hernández \& Carrillo-Salazar, 2012; Suh et al., 2014). It is classified under the Cactacae family with the genus name of Hylocereus. Hylocereus polyrhizus or red-fleshed Pitaya rich in betacyanin pigment is largely cultivated in Indonesia. As people become more interested in natural food, herbal medicines, and traditional practices for a healthy life, the betacyanin pigment from red Pitaya may be utilized as an alternative for dental plaque disclosing agents, while as a food colorant, it has showed promising application in dairy products such as milk and yogurt (Gengatharan, Dykes, \& Choo, 2016; Gengatharan, Dykes, \& Choo 2017).

The objective of this present study was to explore the potential use of extracted red-fleshed Pitaya in various concentrations as a dental plaque disclosing agent in samples taken from volunteers' teeth. A disclosing agent of basic-fuchsin already marketed was used as a positive control.

\section{MATERIALS AND METHODS}

The experimental design and protocols were reviewed and approved by the Ethics Committee in Hasan Sadikin Hospital Bandung, West Java-Indonesia (89/UN6.KEP/EC/2018). All of the subjects gave their informed consent.

\section{Plant material and extract preparation}

Hylocereus polyrhizus fruit were collected in February 2018 from a plantation located in Manglayang, Parongpong, West Bandung Regency, West Java, Indonesia. The fruit was harvested for analysis when it reached the full ripening stage, i.e. 30-35 days after pollination (Figure 1). It was authenticated with collection number 558/HB/02/2018 and deposited in the Herbarium of Plant Taxonomy Laboratory, Department of Biology, Faculty of Math and Nature Sciences, Universitas Padjadjaran Jatinangor, West Java, Indonesia.

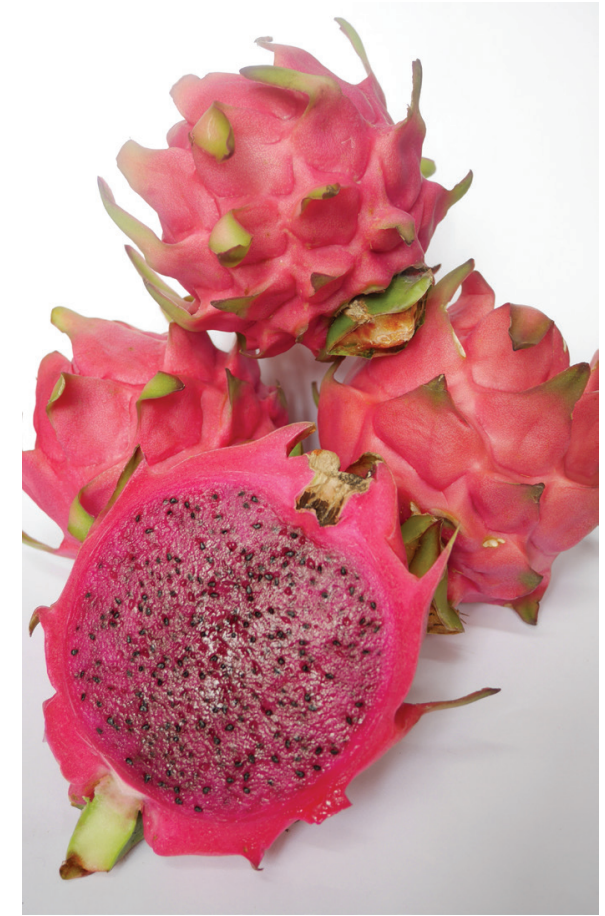

Figure 1. Red-fleshed Pitaya (Hylocereus polyrhizus).

\section{Preparation of Hylocereus polyrhizus flesh extract}

The fruit was washed and wiped dry. Using a stainless-steel knife, the peel was separated from the flesh, and the flesh was cut into portions. The flesh was extracted using distilled water at a fresh weight to solvent ratio $(\mathrm{w} / \mathrm{v})$ of $1: 1$, performed by a maceration process for 72 hours. The extract was then filtrated using filter paper number 41. The solvent was evaporated using a rotary evaporator. The filtrate was diluted into 4 concentrations; 25\%, 50\%, 75\% and 100\%, respectively, subsequently placed in dark glass vials, and stored in a refrigerator until further analysis within 2 days.

\section{Dental plaque samples}

Supragingival dental plaque was harvested from 30 dental students with healthy periodontium and free of caries using a sterile curette. Before plaque sampling, volunteers were given dental prophylaxis and asked to refrain from any dental hygiene measures for 48 hours. To remove the food debris from the tooth surfaces, the volunteers were instructed to rinse with water for 30 seconds before the plaque collection. From each volunteer, one sampling site was chosen from buccal/labial surface, either from molars or incisors representing the 4 regions of the mouth.

Dental plaque was weighted at $1 \mathrm{~g}$ and placed on a sterile glass slide. The specimens were divided into 5 groups and each group was randomly stained either with $0.05 \mathrm{~mL}$ of basic fuchsin (Eviplac, Biodinamica, Brazil) as a positive control, or $25 \%, 50 \%, 75 \%$, or $100 \%$ Pitaya flesh extract. The pigments were absorbed for 60 seconds before being covered and fixed by air-drying and passing through the flame of a Bunsen burner 3 times, with smear side facing up. 


\section{Spectrophotometer analysis}

The specimens were then analyzed using CM 3600D Spectrophotometer (Minolta, Osaka, Japan).

\section{RESULTS}

Thirty samples stained with Pitaya extract were tested to compare color intensity. Color intensity of different concentrations was descriptively presented as mean wavelengths (Table 1).

\section{Table 1. Mean of Color Wavelength assessed by Spectrophotometer}

\begin{tabular}{|lc|}
\hline Agent of Plaque Staining & Wavelength $(\lambda)$ \\
\hline Basic-fuchsin (Eviplac) & 5.9613 \\
Pitaya flesh extract 100\% & 6.4962 \\
Pitaya flesh extract 75\% & 5.3134 \\
Pitaya flesh extract 50\% & 3.3105 \\
Pitaya flesh extract 25\% & 1.8194 \\
\hline
\end{tabular}

Figure 2 presents the comparison of a spectrophotometer curve of positive control from basic-fuchsin staining (green line) with Pitaya extract 25\% (blue line). From the curve, it shows that at $500 \lambda$, basic-fuchsin gives the highest color intensity. From this cut-off point, the color intensity of Pitaya extract 25\% is inferior compared to basic-fuchsin.

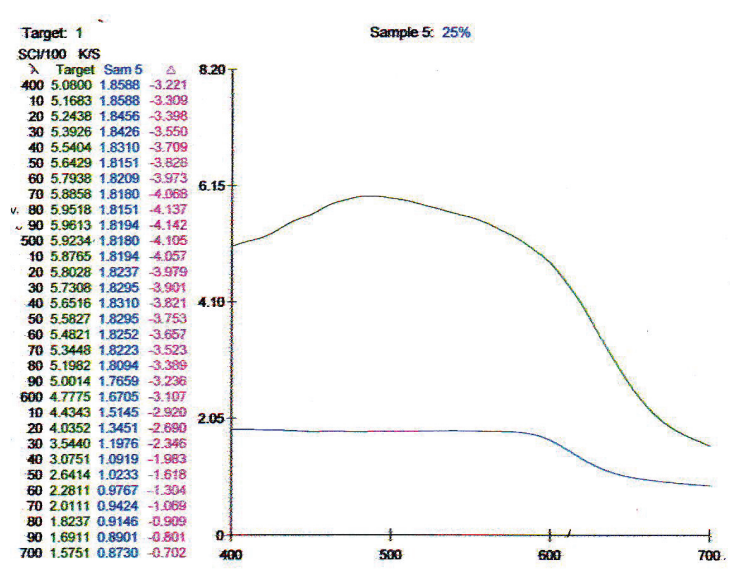

Figure 2. Color wavelength of Pitaya 25\% (blue line) and basic-fuchsin (green line).

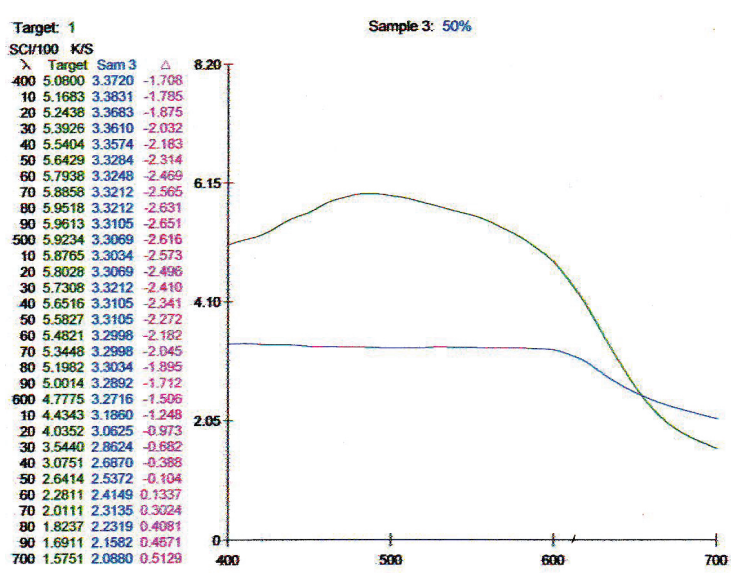

Figure 3. Color wavelength of Pitaya 50\% (blue line) and basic-fuchsin (green line).
Figure 3 is the comparison between positive control (green line) and Pitaya extract 50\% staining (blue line). It shows that Pitaya extract at $50 \%$ color intensity is lower than basic-fuchsin color intensity at $500 \lambda$.

Figure 4 compares positive control (green line) and Pitaya extract at $75 \%$ color intensity (blue line). The color intensity of Pitaya extract is almost the same as basic-fuchsin.

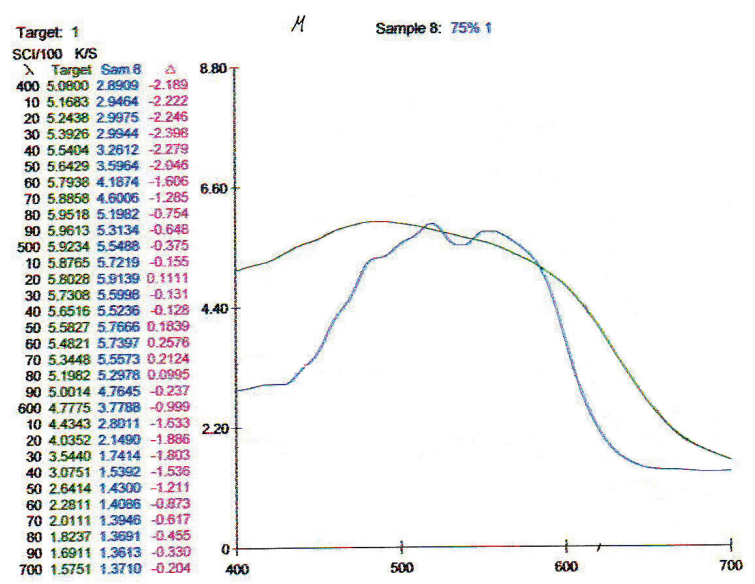

Figure 4. Color wavelength of Pitaya 75\% (blue line) and basic-fuchsin (green line).

Figure 5 exhibits that Pitaya extract at 100\% color intensity is superior to that of basic-fuchsin at $500 \lambda$.

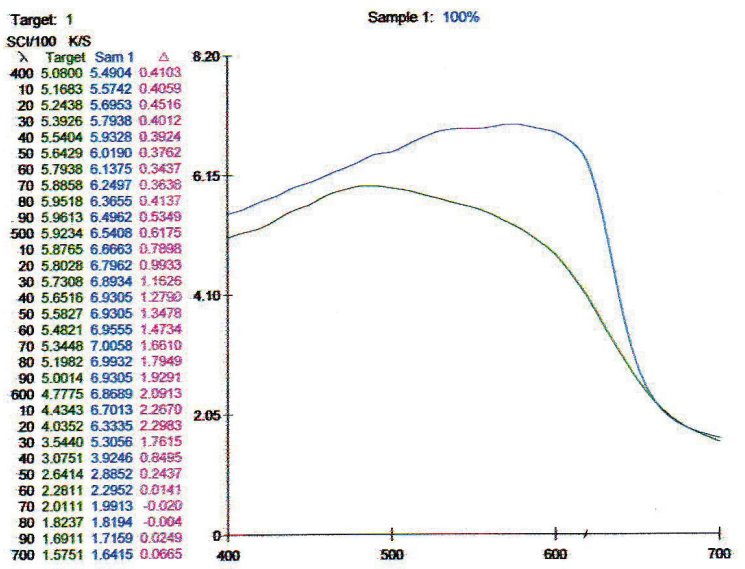

Figure 5. Color wavelength of Pitaya 100\% (blue line) and basic-fuchsin (green line).

Figure 6 shows a stained plaque specimen image

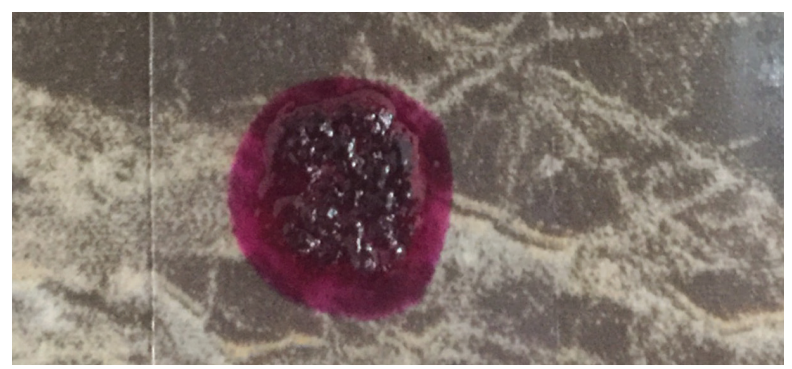

Figure 6. Specimen of dental plaque stained with 75\% Pitaya extract. 
Figure 7 exhibits patient intraoral images of 48-hour old dental plaque without staining

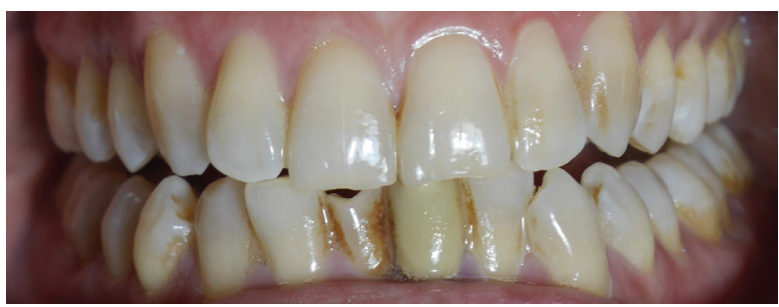

Figure 7. Intra-oral image of dental plaque without staining.

Figure 8 indicates patient intraoral images of 48-hour old dental plaque with staining

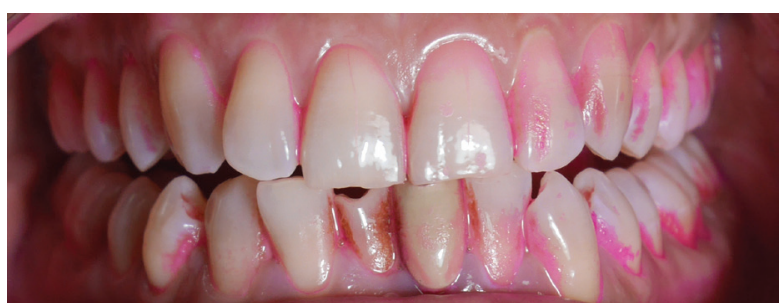

Figure 8. Intra-oral image of dental plaque with staining.

\section{DISCUSSION}

The present study was undertaken to evaluate the potential of using a natural pigment derived from red-fleshed Pitaya fruit extract with different concentrations as a dental plaque disclosing solution.

A disclosing solution is used to reveal plaque to the patient and the clinician to reinforce instruction in oral hygiene. Daily removal of bacterial dental plaque by the patient is paramount to the maintenance of oral health in order to prevent periodontal diseases and tooth decay. Following therapy, these oral hygiene measures play a role in lifelong supportive care (Strömberg, Hagman-Gustafsson, Holmén, Wårdh, \& Gabre, 2012).

Dental plaque has the ability to retain a large number of dye substances because of the polarity difference between the components of the plaque and dyes. The particles are bound to the surface by electrostatic interaction (proteins) and hydrogen bonds (polysaccharides) (Chetrus \& Ion, 2013). Clean tooth surfaces cannot absorb the coloring agent. When pellicle and bacterial plaque are present, they absorb the agent and are revealed. Pellicle stains as a thin relatively clear covering, whereas bacterial plaque appears darker, thicker and more opaque (Sharma, 2010).

In adults with gingivitis, the quality of self-performed mechanical plaque removal is not sufficiently effective and should be improved. Oral hygiene instruction has a significant, albeit small, positive effect on the reduction of gingivitis (Hioe \& van der Weijden, 2005). The existing problem is that total removal of plaque in tooth cleaning measures is difficult to obtain because plaque cannot be seen. Demonstrating the amount of the plaque to patients would improve the standard of oral hygiene. This has been addressed by several research groups. The effect of using plaque disclosing tablets on plaque removal was investigated in subjects refraining from any tooth cleaning for 24 hours; it was shown that significantly more plaque was removed when using the toothbrush together with the disclosing tablet compared to brushing with the toothbrush alone (Sandeep, Vinay, Madhuri, Rao, Uloopi, \& Sekhar, 2014; Peng et al., 2014). In a cross-over study, children without any tooth brushing instruction improved their tooth brushing ability with the use of a disclosing solution staining the amount of dental plaque, as compared with tooth brushing alone (Chounchaisithi, Santiwong, Sutthavong, \& Asvanit, 2014). Moreover, the improved removal of biofilm during periodontal surgery could be accomplished by a clinician, when plaque was revealed by a disclosing solution (Montevecchi, Checchi, Gatto, Klein, \& Checchi, 2012).

The results of the present study showed that Pitaya was able to stain dental plaque with a concentration of $75 \%$ and $100 \%$, which were comparable to the standard disclosing solutions already available on the market. Pitaya fruit produces a colorant of the betacyanin type that belongs to hydro-soluble pigments that presents as deep red-purple, with their stable appearance in a broad pH range. Pitaya fruit have a great potential as natural coloring agents for a wide array of food and are considered to be the third richest betacyanin source for food-coloring agents after Beta vulgaris and Amaranthus species. (Wybraniec \& Mizrahi, 2002). Moreover, betacyanin pigment is also a potent antibacterial agent (Tenore, Novellino, \& Basile, 2012). A disclosing solution is required to have an antibacterial agent; hence Pitaya extract could be a good candidate. It has been proposed that if a disclosing solution has antibacterial properties, then it may be able to protect an open wound in the oral mucosa from unnecessary contamination during a procedure.

Studies of dental plaque staining in vitro with Pitaya extract were scarce, one published study written in Indonesian, observed the penetration strength of staining of flesh extract in potato blocks. The result showed that a concentration of 75\% Pitaya extract had the highest penetration (Hakim, Praharani, \& Purwanto, 2018). Nevertheless, this study was done using visually assessed caliper gauge, that maybe high in risk of bias, despite a calibration performed with 3 examiners. In the present study, a spectrophotometer was employed to analyze the color intensity of plaque staining, eliminating the risk of bias.

Several natural plaque colorants derived from plants have been proposed as plaque disclosing agents. Beta vulgaris (sugar beet), Euterpe oleracea (acai berry) and Bixa orellana (urucum) extracts has been used to stain dental plaque (de Nazaré, Emmi, Barroso, \& da Rocha, 2007). In general, the colorants were prepared by extracting the fruits or the part of a plant by maceration and extracted with a solvent followed by filtration and recuperation of the liquid phase until extracts were obtained.

Betacyanin retention and stability during storage is a crucial factor for the application of a plaque disclosing solution. This present study did not evaluate the degradation of betacyanin of Pitaya extract during the course of the trial. Since light was 
reported previously to accelerate betalain degradation (Herbach, Maier, Stintzing, \& Carle, 2007), the extract was stored in dark storage. Another study showed that refrigeration storage at $4^{\circ} \mathrm{C}$ condition without light exposure managed to preserve the color of the fruit juice up to 3 weeks (Woo, Ngou, Ngo, Soong, \& Tang, 2011). Supplementation with ascorbic acid has been recently reported to delay betacyanin degradation during thermal treatment of purple pitaya juice. The addition of $0.25 \%(w / w)$ ascorbic acid showed a stabilizing effect on betacyanin of concentrate samples that were stored in light. Supplementation with the antioxidant was found to inhibit light damage, thus allowing storage in transparent packaging materials (Wong \& Siow, 2015).

Under the limitation of this study, we concluded that both a $75 \%$ and $100 \%$ extract of pitaya flesh exhibited good dental plaque staining in vitro as assessed by a spectrophotometer. This ability was similar to a benchmark disclosing solution product. This finding merits further study to clarify the optimal concentration and physical stability before its clinical application.

Peer-review: Externally peer-reviewed.

Author Contributions: Conception/Design of Study- A.A., N.R.; Data Acquisition- A.A., R.T.F., N.R.; Data Analysis/Interpretation- A.A., R.T.F.; Drafting Manuscript- A.A.; Critical Revision of Manuscript- A.A.; Final Approval and Accountability- A.A., R.T.F., N.R.; Technical or Material Support- A.A., R.T.F.; Supervision- A.A., N.R.

Conflict of Interest: The authors have no conflict of interest to declare.

Financial Disclosure: Authors declared no financial support.

Acknowledgements: We thank to Textiel Inrichting Bandoeng (Sekolah Tinggi Teknologi Tekstil Bandung) for their assistance in performing spectrophotometry assessment.

\section{REFERENCES}

- Broadbent, J. M., Thomson, W. M., Boyens, J. V., \& Poulton, R. (2011). Dental plaque and oral health during the first 32 years of life. The Journal of the American Dental Association, 142(4), 415-426. https://doi.org/10.14219/jada.archive.2011.0197

- Chetrus, V., \& Ion, I. R. (2013). Dental plaque-classification, formation, and identification. International Journal of Medical Dentistry, 3(2), 139-143.

- Chounchaisithi, N., Santiwong, B., Sutthavong, S., \& Asvanit, P. (2014). Use of a disclosed plaque visualization technique improved the self-performed, tooth brushing ability of primary schoolchildren. Journal of Medical Association Thailand, 97(2), 88-95. Retrieved from https://pdfs.semanticscholar.org/6e3f/92 4e04cade2ae3bdd022a6dc64fab415900a.pdf

- Datta, D., Kumar, S. R., Narayanan, M. A., Selvamary, A. L., \& Sujatha, A. (2017). Disclosing solutions used in dentistry. World Journal of Pharmaceutical Research, 6(6), 1648-1656.

- de Nazaré, R. F. R., Emmi, D. T., Barroso, R. F. F., \& da Rocha, P. O. (2007). U.S. Patent No. 7,182,935. Washington, DC: U.S. Patent and Trademark Office. Retrieved from https://www.lens.org/lens/patent/US_7182935_B2

- Flemmig, T. F., \& Beikler, T. (2011). Control of oral biofilms. Periodontology 2000, 55(1), 9-15. https://doi.org/10.1111/j.16000757.2010.00383.x
Gengatharan, A., Dykes, G. A., \& Choo, W. S. (2016). Stability of betacyanin from red pitahaya (Hylocereus polyrhizus) and its potential application as a natural colourant in milk. International Journal of Food Science \& Technology, 51(2), 427-434. https://doi.org/10.1111/ijfs. 12999

Gengatharan, A., Dykes, G. A., \& Choo, W. S. (2017). The effect of $\mathrm{pH}$ treatment and refrigerated storage on natural colourant preparations (betacyanins) from red pitahaya and their potential application in yoghurt. LWT-Food Science and Technology, 80, 437-445. https://doi.org/10.1016/j.lwt.2017.03.014

Hakim, A., Praharani, D., \& Purwanto, P. (2018). Comparison of Color Penetration between Disclosing Solution and Red Dragon Fruit Extract. (in Indonesian). Proceeding of Seminar : Great Dentist for Achieving Excellent Service, Universitas Jember - East Java, Indonesia. Retrieved from https://repository.unej.ac.id/ handle/123456789/89454

Herbach, K. M., Maier, C., Stintzing, F. C., \& Carle, R. (2007). Effects of processing and storage on juice colour and betacyanin stability of purple pitaya (Hylocereus polyrhizus) juice. European Food Research and Technology, 224(5), 649-658. https://doi.org/10.1007/ s00217-006-0354-5

Hioe, K. P. K. J., \& Van der Weijden, G. A. (2005). The effectiveness of self-performed mechanical plaque control with triclosan containing dentifrices. International Journal of Dental Hygiene, 3(4), 192-204. https://doi.org/10.1111/j.1601-5037.2005.00150.x

Jayanthi, M., Shilpapriya, M., Reddy, V. N., Elangovan, A., Sakthivel, R., \& Vijayakumar, P. (2015). Efficacy of three-tone disclosing agent as an adjunct in caries risk assessment. Contemporary Clinical Dentistry, 6(3), 358. https://dx.doi.org/10.4103\%2F0976-237X.161887 Marsh, P. D. (2010). Microbiology of dental plaque biofilms and their role in oral health and caries. Dental Clinics, 54(3), 441-454. https://doi.org/10.1016/j.cden.2010.03.002

- Miyachi, T., \& Tsutsui, T. (2005). Ability of 13 chemical agents used in dental practice to induce sister-chromatid exchanges in Syrian hamster embryo cells. Odontology, 93(1), 24-29. https://doi. org/10.1007/s10266-005-0055-8

Montevecchi, M., Checchi, V., Gatto, M. R., Klein, S., \& Checchi, L. (2012). The use of a disclosing agent during resective periodontal surgery for improved removal of biofilm. The Open Dentistry Journal, 6, 46-50. https://dx.doi.org/10.2174\% 2F1874210601206010046

Ortiz-Hernández, Y. D., \& Carrillo-Salazar, J. A. (2012). Pitahaya (Hylocereus spp.): a short review. Comunicata Scientiae, 3(4), 220-237. https://doi.org/10.14295/cs.v3i4.334

Peng, Y., Wu, R., Qu, W., Wu, W., Chen, J., Fang, J. ... \& Mei, L. (2014). Effect of visual method vs plaque disclosure in enhancing oral hygiene in adolescents and young adults: A single-blind randomized controlled trial. American Journal of Orthodontics and Dentofacial Orthopedics, 145(3), 280-286. https://doi.org/10.1016/j. ajodo.2013.10.021

- Sandeep, V., Vinay, C., Madhuri, V., Rao, V. V., Uloopi, K. S., \& Sekhar, R. C. (2014). Impact of visual instruction on oral hygiene status of children with hearing impairment. Journal of Indian Society of Pedodontics and Preventive Dentistry, 32(1), 39-43. https://doi. org/10.4103/0970-4388.127053

Sania, M. S., Aggarwal, M.C., \& Chaubey, K. K. (2016) An overreaction to plaque disclosing agent: an unusual case. Journal of Periodontal Medicine and Clinical Practice, 3, 51-55.

- $\quad$ Seneviratne, C. J., Zhang, C. F., \& Samaranayake, L. P. (2011). Dental plaque biofilm in oral health and disease. Chinese Journal of Dental Research, 14(2), 87-94.

Sharma, S. (2010). Plaque disclosing agent a review. Journal of Advanced Dental Research, 2(1). Available from: http://www.ispcd. org/ cmsdev/userfi les/ rishabh/plaque\%20fi nal\%20new.pdf. 
- Strömberg, E., Hagman-Gustafsson, M. L., Holmén, A., Wårdh, I., \& Gabre, P. (2012). Oral status, oral hygiene habits and caries risk factors in home-dwelling elderly dependent on moderate or substantial supportive care for daily living. Community Dentistry and Oral Epidemiology, 40(3), 221-229. https://doi.org/10.1111/ j.1600-0528.2011.00653.X

- $\quad$ Suh, D. H., Lee, S., Heo, D. Y., Kim, Y. S., Cho, S. K., Lee, S., \& Lee, C. H. (2014). Metabolite profiling of red and white pitayas (Hylocereus polyrhizus and Hylocereus undatus) for comparing betalain biosynthesis and antioxidant activity. Journal of Agricultural and Food Chemistry, 62(34), 8764-8771. https://doi.org/10.1021/jf5020704

- Tenore, G. C., Novellino, E., \& Basile, A. (2012). Nutraceutical potential and antioxidant benefits of red pitaya (Hylocereus polyrhizus) extracts. Journal of Functional Foods, 4(1), 129-136. https://doi. org/10.1016/j.jff.2011.09.003
Wong, Y. M., \& Siow, L. F. (2015). Effects of heat, pH, antioxidant, agitation and light on betacyanin stability using red-fleshed dragon fruit (Hylocereus polyrhizus) juice and concentrate as models. Journal of Food Science and Technology, 52(5), 3086-3092. https://doi.org/10.1007/s13197-014-1362-2

- Woo, K. K., Ngou, F. H., Ngo, L. S., Soong, W. K., \& Tang, P. Y. (2011). Stability of betalain pigment from red dragon fruit (Hylocereus polyrhizus). American Journal of Food Technology, 6(2), 140-148. Retrieved from http://docsdrive.com/pdfs/academicjournals/ ajft/2011/140-148.pdf

- Wybraniec, S., \& Mizrahi, Y. (2002). Fruit flesh betacyanin pigments in Hylocereus cacti. Journal of Agricultural and Food Chemistry, 50(21), 6086-6089. https://doi.org/10.1021/jf020145k 